\title{
Generation of continuous and pulsed diagnostic imaging $x$-ray radiation using a carbon-nanotube-based field-emission cathode
}

\author{
G. Z. Yue \\ Department of Physics, University of North Carolina, Chapel Hill, North Carolina 27599 \\ Q. Qiu and Bo Gao \\ Applied Nanotechnologies, Inc., 308 West Rosemary Street, Chapel Hill, North Carolina 27516 \\ Y. Cheng, J. Zhang, and H. Shimoda \\ Department of Physics and Astronomy, University of North Carolina, Chapel Hill, North Carolina 27599 \\ S. Chang \\ Department of Radiation Oncology, School of Medicine, University of North Carolina, \\ Chapel Hill, North Carolina 27599 \\ J. P. Lu and O. Zhou ${ }^{\text {a) }}$ \\ Department of Physics and Astronomy and Curriculum in Applied and Materials Sciences, \\ University of North Carolina, Chapel Hill, North Carolina 27599
}

(Received 12 April 2002; accepted for publication 15 May 2002)

\begin{abstract}
$\mathrm{X}$-ray radiation is widely used in medical and industrial applications. The basic design of the x-ray tube has not changed significantly in the last century. In this paper, we demonstrate that medical diagnostic x-ray radiation can be generated using a carbon nanotube (CNT)-based field-emission cathode. The device can readily produce both continuous and pulsed x-rays with a programmable wave form and repetition rate. A total emission current of $28 \mathrm{~mA}$ was obtained from a $0.2 \mathrm{~cm}^{2}$ area CNT cathode. The $\mathrm{x}$-ray intensity is sufficient to image a human extremity at $14 \mathrm{kVp}$ and $180 \mathrm{mAs}$. Pulsed x-ray with a repetition rate greater than $100 \mathrm{kHz}$ was readily achieved by programming the gate voltage. The CNT-based cold-cathode x-ray technology can potentially lead to portable and miniature x-ray sources for industrial and medical applications. (C) 2002 American Institute of Physics. [DOI: 10.1063/1.1492305]
\end{abstract}

A conventional $\mathrm{x}$-ray tube is comprised of a metal filament (cathode) that emits electrons when resistively heated to over $1000{ }^{\circ} \mathrm{C}$ and a metal target (anode) that emits $\mathrm{x}$ rays when bombarded by the accelerated electrons. ${ }^{1}$ The intensity of the $\mathrm{x}$-ray radiation is proportional to the electron current and the square of the acceleration voltage. This design has several inherent limitations. Thermionic cathodes in general have a slow response time and high power consumption. The high operating temperature reduces the lifetime of the $\mathrm{x}$-ray tubes due to failure of the metal filament. It is estimated that the average life time of an x-ray tube in a typical x-ray machine is less than a year, and a large portion of $\mathrm{x}$-ray tube failures in diagnostic x-ray units are filament related. In addition, the emitted electrons are randomly distributed. Although they can be focused by a bias electrical field, the resulting beam has a non-Gaussian intensity distribution, which limits the imaging resolution.

Field emission is a much more attractive mechanism to extract electrons compared to thermionic emission because electrons are emitted at room temperature and the output current is voltage controllable. ${ }^{2}$ Field emission cathodes have found applications in a variety of vacuum electronic devices, such as field emission displays and electron guns in the microscope. ${ }^{3}$ Although the concept of cold-cathode x-ray tubes has been investigated in the past using materials in-

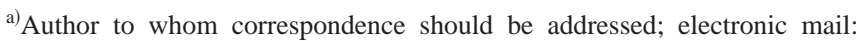
zhou@physics.unc.edu
}

cluding diamond and carbon nanotubes (CNTs), ${ }^{4-7}$ development has been hindered by the lack of cathodes that can deliver stable currents comparable to the values used in conventional $\mathrm{x}$-ray tubes (10-50 mA for fixed anode and 50$500 \mathrm{~mA}$ for rotating anode tubes). Diamond emitters are in general unstable at current densities higher than $30 \mathrm{~mA} / \mathrm{cm}^{2}$. CNTs can potentially produce a much higher emission current. It is found experimentally that an individual CNT can emit $\sim 1 \mu \mathrm{A}$ per tube. ${ }^{8}$ However, the current density decreases drastically with increasing emission area due to problems such as uniformity. As a result, high field-emission current has not been obtained.

By optimization of the morphology of CNT films, we have recently demonstrated large and stable emission current from macroscopic CNT cathodes. ${ }^{9,10}$ Here we report the performance of these cathodes in a triode-type field-emission $\mathrm{x}$-ray tube. The results show that this type of tube can generate $\mathrm{x}$-ray flux comparable to that from the conventional fixed-target $\mathrm{x}$-ray tubes, and can produce high frequency pulsed x-ray radiation with programmable width and repetition.

The emission material used in this study is purified single-wall carbon nanotube (SWNT) bundles that were produced by the laser ablation method ${ }^{11}$ at UNC. ${ }^{12}$ They contain $\sim 95$ wt \% SWNT bundles with the average tube diameter of $1.4 \mathrm{~nm}$ and bundle diameter of $\sim 50 \mathrm{~nm}$. A uniform layer of SWNTs was coated on a flat metal disc by electrophoretic deposition. ${ }^{10}$ To increase the adhesion between the SWNTs 

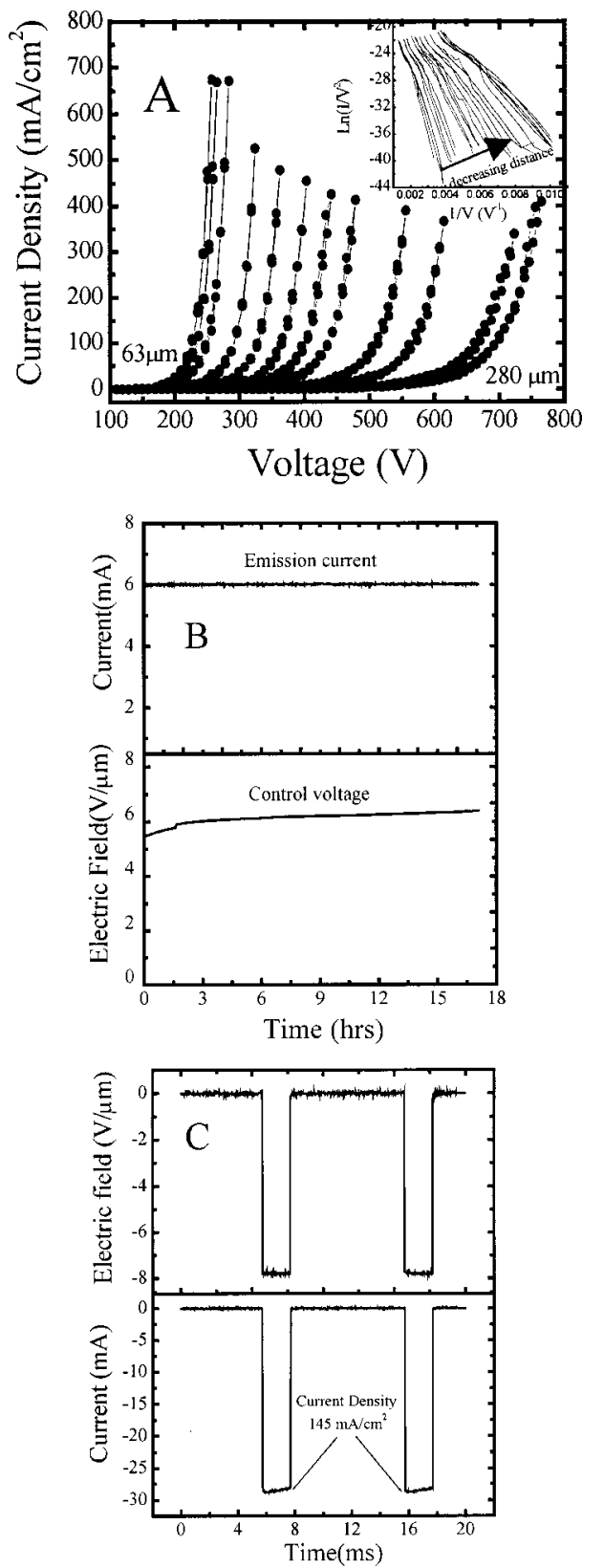

FIG. 1. (a) Electron emission current vs applied voltage of a SWNT film measured at different anode-cathode distances using a hemispherical anode ( $1.0 \mathrm{~mm}$ in diameter). The threshold field for $1 \mathrm{~mA} / \mathrm{cm}^{2}$ is $2 \mathrm{~V} / \mu \mathrm{m}$. Inset is the same set of data plotted as $\ln \left(I / V^{2}\right)$ vs $1 / V$ (Fowler-Nordheim plot) which show linear dependence. (b) Applied voltage vs time when the total emission current was set at $6 \mathrm{~mA}(10 \%$ duty cycle). The emission current was measured under the parallel plate geometry. The applied voltage was automatically adjusted to maintain the set current via a feedback system. (c) Total emission current from a $0.2 \mathrm{~cm}^{2}$ area SWNT film measured under the pulsed mode. The pulsed width and repetition rate were controlled by programming the gate voltage. A peak current of $28 \mathrm{~mA}$ was obtained without breakdown.

and the substrate, an iron interlayer was first deposited on the substrate by either thermal evaporation or electrochemical plating before nanotube deposition. ${ }^{13}$ The thickness and packing density of the nanotube film were controlled by the current, deposition time and the concentration of the nanotube suspension.

The emission current-voltage $(I-V)$ characteristics of the SWNT film were measured using a hemispherical current collector (anode) with a 1-mm-diam tip at $5 \times 10^{-7}$ Torr base pressure at different anode-cathode gap distances. As
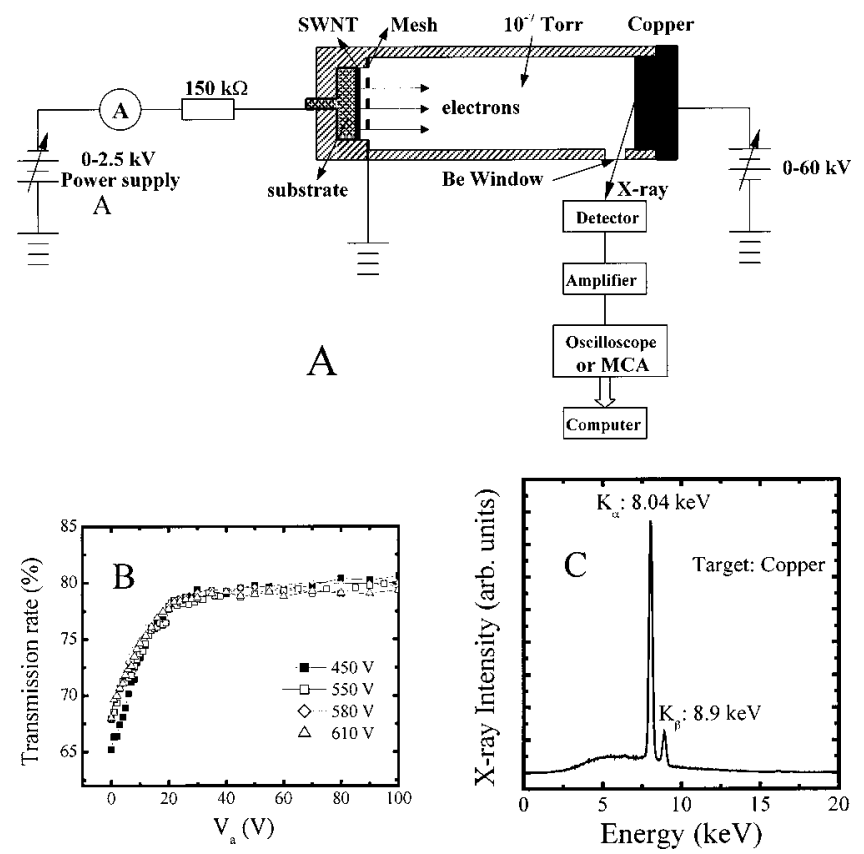

FIG. 2. (a) A schematic of the triode-type field emission x-ray tube. SWNTs coated on a metal substrate were used as the cathode. The gate electrode is a metal mesh 50-200 $\mu \mathrm{m}$ away from the cathode. Electron emission is triggered by the voltage applied between the gate and the cathode. X-ray is produced when the emitted electrons were accelerated and bombarded on the copper target. (b) The emission current transmission rate $I_{a} /\left(I_{a}+I_{g}\right)$ versus anode voltage $\left(V_{a}\right)$ measured in the triode configuration at different gate voltages. By adjusting the distance between the gate and the cathode and the opening of the metal grids, a transmission rate of $80 \%$ (current gain $I_{a} / I_{g}$ of 4) was obtained at $V_{a}>50 \mathrm{~V}$. (c) Energy spectrum of the x ray generated from a copper target at an acceleration voltage of $14 \mathrm{kV}$. The data was recorded by a Si-PIN photodiode $\mathrm{x}$-ray detector and a multichannel analyzer.

shown in Fig. 1(a) and inset, the sample shows the classic Fowler-Nordheim behavior with a threshold field of $2 \mathrm{~V} / \mu \mathrm{m}$ for $1 \mathrm{~mA} / \mathrm{cm}^{2}$ current density (the effective emission area was calculated using a previously described method ${ }^{14}$ ). Emission current density over $1 \mathrm{~A} / \mathrm{cm}^{2}$ was readily achieved.

The total emission current from the macroscopic cathode $\left(0.2 \mathrm{~cm}^{2}\right)$ was measured using the parallel-plate geometry. No overall decay of the emission current was observed over a 10-h-period at $2 \mathrm{~mA}\left(10 \mathrm{~mA} / \mathrm{cm}^{2}\right)$ under a constant $\mathrm{dc}$ voltage without feedback (after the initial "burn-in"). The standard deviation of the current fluctuation is $2 \%-4 \%$. The fluctuation and stability can be improved significantly by incorporation of a simple feedback loop, ${ }^{9}$ as demonstrated in Fig. 1(b). Here the total emission current was fixed at $6 \mathrm{~mA}$ $\left(10 \%\right.$ duty cycle, $\left.30 \mathrm{~mA} / \mathrm{cm}^{2}\right)$ and the applied voltage was adjusted automatically. High frequency pulsed emission with a variable pulse width and repetition rate can be readily obtained by programming the applied electrical field. Figure 1 (c) shows the pulsed emission current generated using a 100 $\mathrm{Hz}$ and 2-ms-width square wave. A peak emission current of $28 \mathrm{~mA}$ was obtained from a $0.2 \mathrm{~cm}^{2}$ SWNT film (140 $\mathrm{mA} / \mathrm{cm}^{2}$ ). This emission current, to our best knowledge, is an order of magnitude higher than the values previously reported from macroscopic cathodes.

The performance of the SWNT film as a cold-cathode for x-ray generation was tested using the triode geometry. As shown in Fig. 2(a), the experimental setup consists of a gated SWNT field-emission cathode and a copper metal target to AIP license or copyright, see http://ojps.aip.org/aplo/aplcr.jsp 


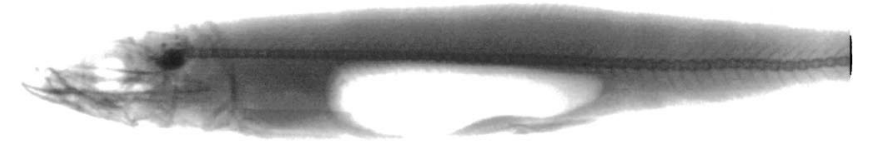

A

B

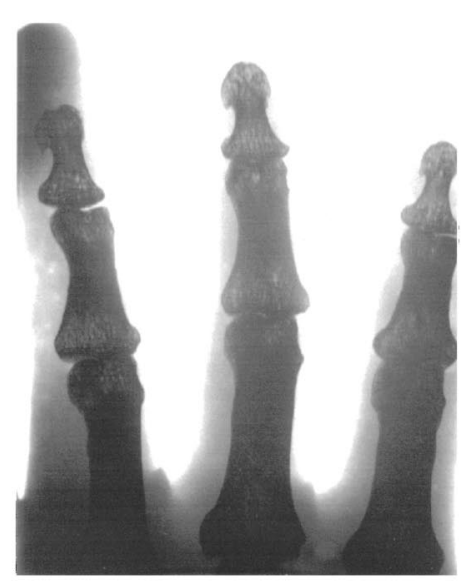

C

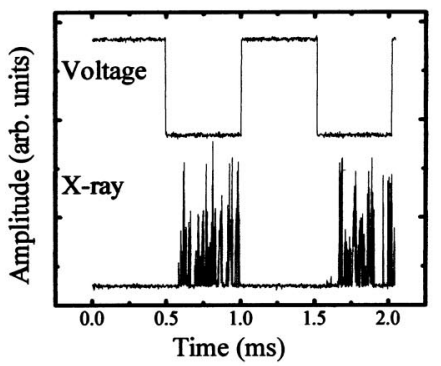

FIG. 3. X-ray images of a fish (A) and a humanoid hand (B) taken using Polaroid ${ }^{\mathrm{TM}}$ films placed behind the objects which were $30 \mathrm{~cm}$ away from the x-ray source. The exposure condition was $14 \mathrm{kV} p$ and $180 \mathrm{mAs}$. Detailed bone structures are clearly resolved. c) $1 \mathrm{kHz}$ and $50 \%$ duty cycle pulsed $\mathrm{x}$-ray radiation generated by applying a pulse gate voltage with the same wave form. The $\mathrm{x}$-ray signals were recorded using an oscilloscope. The height of the signal indicates the photon energy rather than the intensity. The $\sim 100 \mu$ s delay between the onset of $V_{g}$ and x-ray emission can be eliminated by reducing the in-line resistance used (and thus the RC time constant). The wave form and the repetition rate of the $\mathrm{x}$-ray can be easily programmed. A $100 \mathrm{kHz}$ x-ray was generated by the current setup, which is limited by the response time of the $\mathrm{x}$-ray detector.

housed in vacuum chamber at $10^{-6}$ Torr base pressure. The gate, a metal grid 50-200 $\mu \mathrm{m}$ away from the cathode, was grounded. A negative voltage was applied to the cathode $\left(V_{g}\right)$ and a positive voltage to the anode $\left(V_{a}\right)$. First, electron emission of the triode structure was characterized. The total emission $(I)$, anode $\left(I_{a}\right)$, and the gate $\left(I_{g}\right)$ currents were measured versus $V_{g}$ and $V_{a}$. After optimizing the geometry of the grid, a current transmission rate $I_{a} /\left(I_{a}+I_{g}\right)$ of $80 \%$ (current gain $I_{a} / I_{g}$ of 4) was obtained at $V_{a}>50 \mathrm{~V}$ [Fig. 2(b)]. The high transmission rate is important for minimizing overheating of the gate during operation.

For x-ray generation, field-emitted electrons were accelerated by the anode voltage $V_{a}$ to bombard on the $\mathrm{Cu}$ target. A Si-PIN photodiode detector and a multichannel analyzer were used to record the data. As expected, the energy spectrum of the $\mathrm{x}$ ray thus generated is the same as those by the thermionic electrons, with strong characteristic $\mathrm{Cu} K_{\alpha}$ and $\mathrm{K}_{\beta}$ lines and a broad Bremsstrahlung background [Fig. 2(c)]. The x-ray intensity is proportional to $I_{a}$ and $V_{a}^{2}$, which can

$\mathbf{7 5}, 873$ (1999).
be independently controlled in the triode mode.
Downloaded 06 Nov 2002 to 152.2.6.144. Redistribution subject to AIP license or copyright, see http://ojps.aip.org/aplo/aplcr.jsp
To demonstrate the viability of this CNT based fieldemission X-ray source, images of a fish and a humanoid hand were taken using Polaroid ${ }^{\mathrm{TM}}$ films placed behind the objects outside the x-ray chamber. Due to the limitation of the present power supply and vacuum feedthrough, the acceleration voltage was set at $14 \mathrm{kV}$ which is substantially lower than the 50-100 kV value commonly used for medical imaging. ${ }^{1}$ The effective focal spot of the $\mathrm{x}$-ray source is about $3.2 \mathrm{~mm}$ in diameter. The distance between the source and the object is $30 \mathrm{~cm}$. As shown in Fig. 3 fine structures of the humanoid hand and fish were clearly resolved.

High frequency and high-intensity pulsed $\mathrm{x}$ ray is desirable for both medical and industrial applications. By replacing the dc gate voltage with a pulsed signal, pulsed $\mathrm{x}$ ray with programmable width and repetition rate was easily produced using the device described here. Figure 3(c) demonstrates $1 \mathrm{kHz}$ and $50 \%$ duty cycle pulsed $\mathrm{x}$ ray generated by applying a square wave form $V_{g}$. A $\sim 100 \mu$ s delay between the onset of $V_{g}$ and x-ray photon was observed. This is due to the capacitance and the in-line resistor used for overcurrent protection in the electrical circuitry $(C=293 \mathrm{pF}$ and $R$ $=160 \mathrm{k} \Omega, \tau=47 \mu \mathrm{s}$ ), and can be essentially eliminated by reducing the resistance. By replacing the gap between the gate the cathode to the micron range, pulsation can be triggered by an off-the-shelf signal generator without further amplification.

The cold-cathode $\mathrm{x}$-ray tube demonstrated here overcomes several limitations of the conventional x-ray tubes. By elimination of the resistively heated metal filaments the life span of $x$-ray tubes can be greatly prolonged. The size of the $\mathrm{x}$-ray tube and its power supply can also be reduced significantly to produce portable and miniature x-ray machines. Carbon nanotube field-emission cathodes also have the ability to produce focused electron beams with a small energy spread that can potentially enable $\mathrm{x}$-ray tubes with ultrafine focal spots for high-resolution imaging.

We thank S. Shafroth, T. Clegg, and N. Parikh for discussion and assistance. This work was partially supported by the Office of Naval Research under a MURI program (N00014-98-1-0597) at UNC and a grant from Applied Nanotechnologies, Inc.

${ }^{1}$ S. C. Bushong, Radiologic Science for Technologist (Mosby, St. Louis, 1997).

${ }^{2}$ I. Brodie and C. A. Spindt, Adv. Electron. Electron Phys. 83, 1106 (1992).

${ }^{3}$ Vacuum Micro-electronics, edited by W. Zhu (Wiley, New York, 2001).

${ }^{4}$ R. R. Whitlock, M. I. Bell, D. V. Kerns, S. Kerns, J. L. Davidson, and W. P. Kang, US Patent No. 6,333,968 (2001).

${ }^{5}$ R. Baptist, US Patent No. 6,259,765 (2001).

${ }^{6}$ P. Rangstein, C. Ribbing, C. Strandman, R. Hok, and L. Smith, Sens. Actuators 82, 24 (2000).

${ }^{7}$ H. Sugie, M. Tanemura, V. Filip, K. Iwata, K. Takahashi, and F. Okuyama, Appl. Phys. Lett. 78, 2578 (2001).

${ }^{8}$ K. A. Dean and B. R. Chalamala, Appl. Phys. Lett. 76, 375 (2000).

${ }^{9}$ G. Z. Yue, B. Gao, Y. Cheng, J. Zhang, H. Shimoda, and O. Zhou (unpublished)

${ }^{10}$ B. Gao, G. Z. Yue, Q. Qiu, Y. Cheng, H. Shimoda, L. Fleming, and O. Zhou, Adv. Mater. 13, 1770 (2001).

${ }^{11}$ A. Thess, R. Lee, P. Nikdaev, H. Dai, P. Petit, J. Robert, C. Xu, Y. H. Lee, S. G. Kim, A. G. Rinzler, D. T. Colbert, G. E. Scuseria, D. Tomanek, J. E. Fischer, and R. E. Smalley, Science 273, 483 (1996).

${ }^{12}$ X. P. Tang, A. Kleinhammes, H. Shimoda, L. Fleming, C. Bower, S. Sinha, O. Zhou, and Y. Wu, Science 228, 492 (2000).

${ }^{13}$ C. Bower, O. Zhou, and W. Zhu, US Patent No. 6,277,318 (2001)

${ }^{14}$ W. Zhu, C. Bower, O. Zhou, G. P. Kochanski, and S. Jin, Appl. Phys. Lett.

75, 873 (1999).
AIP license or copyright, see http://ojps.aip.org/aplo/aplcr.jsp 